\title{
Periodontal disease exacerbates systemic ovariectomy-induced bone loss in mice
}

\author{
Ana Lia Anbinder ${ }^{\mathrm{a}, *}$, Renata M. Moraes ${ }^{\mathrm{a}}$, Gabriela M.G. Lima ${ }^{\mathrm{a}}$, Felipe E. Oliveira ${ }^{\text {a }}$, Débora R.C. Campos ${ }^{\mathrm{a}}$, \\ Rodnei D. Rossoni ${ }^{\text {a }}$, Luciane D. Oliveira ${ }^{\text {a }}$, Juliana C. Junqueira ${ }^{\text {a }}$, Yun Ma ${ }^{\text {b }}$, Florent Elefteriou ${ }^{\text {b, } 1}$ \\ a Department of Bioscience and Oral Diagnosis, Institute of Science and Technology of São José dos Campos, Univ Estadual Paulista-UNESP, São José dos Campos, São Paulo, Brazil

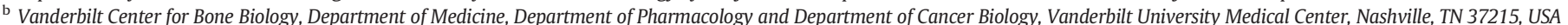

\section{A R T I C L E I N F O}

Article history:

Received 4 October 2015

Revised 20 November 2015

Accepted 21 November 2015

Available online 24 November 2015

\section{Keywords:}

Periodontitis

Osteoporosis

Fracture risk

TNF- $\alpha$

Ovariectomy

\begin{abstract}
A B S T R A C T
Periodontal pathogens and/or inflammatory products from periodontitis participate in the development or progression of systemic diseases. In this context, periodontitis acts as a modifying factor to systemic health, including diabetes and cardiovascular diseases. Osteoporosis is an increasingly prevalent condition in our aging population and considered a risk factor for periodontal disease, but the effect of periodontitis on systemic bone homeostasis is unknown. We thus evaluated the effects of experimental periodontitis (EP) on systemic bone loss and the influence of estrogen deficiency in this context, using a mouse model of combined periodontitis and osteoporosis. Experimental periodontitis (EP) was induced by a ligature insertion around the mandibular first molars and Porphyromonas gingivalis infection. Three-dimensional microcomputed tomographic analyses performed 48 days following infection revealed that EP and ovariectomy (OVX) induced a significantly higher femoral and mandibular bone loss compared to EP or OVX alone. EP alone did not induce systemic bone loss. In addition, the EP + OVX and EP groups showed significantly higher levels of tumor necrosis factor (TNF)- $\alpha$ than OVX and control groups at end point. These results suggest that periodontitis could be a risk factor for systemic bone loss, especially in post-menopausal women, and warrant further clinical investigations to confirm this association and propose adapted prophylactic and curative therapies.
\end{abstract}

(c) 2015 Elsevier Inc. All rights reserved.

\section{Introduction}

The world population is aging and the proportion of individuals over 65 is increasing [1]. Considering that menopause occurs around the age of 50 , millions of women will live many years at risk for osteoporosis and fractures [2].

Osteoporosis is a systemic skeletal disease characterized by low bone density and microarchitectural deterioration of bone tissues, which leads to increased bone fragility and risk of fracture [3]. The influence of osteoporotic fractures in public health includes effects on mortality, functional impairment, quality of life and has a costly financial impact [4].

Periodontitis can be defined as an inflammation of the support tissue around the teeth, leading to local bone tissue loss and destruction of the periodontal ligament, or as an extension of gingivitis to adjacent bone and ligament tissues [5]. From 2009 to 2012, 46\% of adults in the US,

\footnotetext{
* Corresponding author at: Department of Bioscience and Oral Diagnosis, Institute of Science and Technology of São José dos Campos, Univ Estadual Paulista-UNESP, Av Engenheiro Francisco José Longo, 777, São José dos Campos, São Paulo CEP: 12.245-000, Brazil.

E-mail address: ana.anbinder@fosjc.unesp.br (A.L. Anbinder).

${ }^{1}$ Present address: Baylor College of Medicine, Department of Molecular and Human Genetics and Orthopedic Surgery, One Baylor Plaza, Houston, TX 77030, USA.
}

representing 64.7 million people, had periodontitis, with $8.9 \%$ having severe periodontitis [6].

Osteoporosis and periodontitis are inflammatory diseases characterized by bone resorption. Both are silent diseases, being asymptomatic until late stages, and share several risk factors, such as tobacco use and advanced age [7]. Initiation and progression of periodontal disease can be modified by local factors and systemic conditions. Systemic risk factors include gender, smoking and alcohol, as well as medical conditions such as poorly controlled diabetes, obesity, stress and inadequate dietary consumption of calcium and vitamin D [8]. Although osteoporosis has also been considered a risk factor for the progression of periodontal disease $[7,9,10]$, this relationship is controversial $[10,11]$.

Periodontal pathogens and/or inflammatory products from periodontitis participate in the development or progression of systemic diseases [12]. In this context, periodontitis acts as a modifying factor to systemic health, including vascular disorders associated with atherosclerosis, diabetes mellitus, respiratory disorders, as well as several debated associations such as preterm delivery, rheumatoid arthritis, pancreatic cancer and metabolic syndrome [12]. Although a growing body of literature has accumulated to investigate osteoporosis as risk factor for periodontitis [7,9-11], the inverse interaction, i.e., the effect of periodontitis on systemic bone remodeling, is unclear [11,12]. Interestingly, older individuals with both osteoporosis and periodontitis 
are at greater risk for future fractures than those with either osteoporosis or periodontitis alone [13]. This observation led us to hypothesize that periodontitis is a risk factor for systemic bone loss and to evaluate the effects of experimental periodontitis (EP) on ovariectomy-induced systemic bone loss in mice.

There are several well-established models to induce EP in rats and mice, such as the insertion of a ligature around molars [14,15], bacterial infection by oral gavage [15-17], lipopolysaccharide injection [15], or association between ligature and bacterial infection [18-20]. The modalities and the duration of periodontitis induction are very important, since they influence periodontal tissue destruction and serum levels of proinflammatory cytokines as well, and different protocols can lead to diverse results [19]. The ligature model has several disadvantages related to the trauma caused during the ligature insertion and to the decrease in disease severity with time [18]. The oral gavage model on the other hand, first reported by Baker et al. [16], does not induce an acute alveolar bone loss, as opposed to the ligature insertion $[15,18]$. Ligature associated to Porphyromonas gingivalis infection generated more active periodontitis [19], with increased periodontal bone resorption [21], more intense systemic response than ligature or oral $P$. gingivalis infection alone [19] and also led to the maintenance of the disease intensity with time [18]. Therefore, ligature associated to $P$. gingivalis infection was used to experimentally address our hypothesis in mice.

\section{Material and methods}

Animal care and study protocols were approved by the Institute of Science and Technology of São José dos Campos (UNESP) Ethics Committee (06/2012-PA/CEP). Forty-five 8-week-old specific pathogen free female mice (Mus musculus, $B A L B / c$ ), approximately $25 \mathrm{~g}$, were housed in a light- and temperature-controlled environment (12-hlight: 12-h-dark cycle; $22 \pm 2{ }^{\circ} \mathrm{C}$ ). After a 2-week adaptation, they were randomly divided into four groups, as follows: 1) OVX group: ovariectomy (OVX) without EP induction ( $\mathrm{n}=13) ; 2$ ) Control group: OVX-sham surgery without EP induction $(\mathrm{n}=10) ; 3)$ OVX + EP group: EP and ovariectomy $(\mathrm{n}=10)$; 4$)$ EP group: induced EP and OVX-sham surgery $(\mathrm{n}=$ 12).

Standard pellet diet was offered ad libitum to mice submitted to sham surgery, and ovariectomized animals were pair fed, i.e., restricted to the average amount of food eaten by the sham ones. We measured the daily food intake of sham mice by weighing the amount of food remaining in each cage every $24 \mathrm{~h}$, for two weeks. All animals received water ad libitum. Body weight was measured at the day of OVX/Sham surgery and at euthanasia and these values were converted to percentage of body weight gain.

\subsection{Ovariectomy and sham surgery}

All animals were anesthetized using injection of ketamine base (100-150 mg/kg)/xylazine hydrochloride (10-15 mg/kg), and submitted to ovariectomy or sham surgery on day 0 .

Bilateral ovariectomy was performed following a bilateral longitudinal skin incision in the lateral abdominal region. Sham surgery was performed by exposing the ovaries without excision. Ovariectomy was performed by exposing the ovaries and excision with an electrocautery instrument. The peritoneal muscle was sutured with an absorbable suture thread (catgut 4-0, Ethicon, Johnson \& Johnson, São José dos Campos, SP, Brazil) and skin with a silk line (3-0, Ethicon, Johnson \& Johnson, São José dos Campos, SP, Brazil). Efficacy of ovariectomy was confirmed by bilateral uterus atrophy at euthanasia.

\subsection{Bacterial strains and culture}

P. gingivalis (ATCC 33277 ) was cultured at $37^{\circ} \mathrm{C}$ on Fastidious Anaerobe Agar (Acumedia, Lansing, MI, USA) plates with sheep blood, supplemented with $0.1 \%$ menadione and hemin (Sigma-Aldrich, Saint Louis, MO, USA) in an oxygen-free atmosphere (80\% nitrogen, $10 \%$ carbon dioxide and $10 \%$ hydrogen), as described previously [22]. After 7 days of growth on blood agar, $P$. gingivalis colonies were selected, and a solution of $10^{9} \mathrm{CFU} / \mathrm{ml}$ in $100 \mu \mathrm{l}$ of $2 \%$ carboxymethylcellulose [16](Sigma-Aldrich, Saint Louis, MO, USA) was prepared. This inoculum was prepared immediately before use.

\subsection{Periodontal disease induction}

Periodontal disease was induced by the insertion of a ligature around the lower first molar associated to $P$. gingivalis infection.

All mice were treated with sulfamethoxazole-trimethoprim (Bactrim-Roche Químicos e Farmacêuticos S.A, Rio de Janeiro, RJ, Brazil) $(10 \mathrm{ml} / \mathrm{l})$ in water, ad libitum for 10 days (day-11 to day-1; Fig. 1) to reduce native flora [16]. This was followed by a 3-day antibioticfree period [16](Fig. 1). On day 3, animals from EP + OVX and EP groups were anesthetized again before the insertion of the ligature. A 4-0 silk thread (Ethicon, Johnson \& Johnson, São José dos Campos, SP, Brazil) was wrapped around the first inferior molars of the animals, carefully pushing the ligature into the gingival sulcus and knotting mesially. Periodontal disease was not induced in the animals of the OVX and control groups, however, they were anesthetized and manipulated in the same way as the others.

Animals from OVX + EP and EP groups were infected with $P$. gingivalis $\left(10^{9} \mathrm{CFU}\right.$ in $100 \mu \mathrm{l}$ of phosphate-buffered saline with $2 \%$ of carboxymethylcellulose) [16] by gavage ( $50 \mu \mathrm{l}$ placed into the esophagus and $50 \mu \mathrm{l}$ into oral cavity) eight times [17] at 2-day intervals (days $3,6,9,12,15,18,21$, and 25; Fig. 1). Control and OVX mice received the carboxymethylcellulose gavage without $P$. gingivalis.

On days 18 and 33, the animals were anesthetized, the presence of the ligature was evaluated (groups EP and OVX $+\mathrm{EP}$ ), and the animals in which it was absent were excluded from the study. At this time, we repositioned the thread in an apical position to maintain the ligature in intimate contact with the marginal tissues.

\subsection{Euthanasia}

Euthanasia was performed on day 51 ( 51 days after the day of OVX or sham surgery) [23]. Animals were anesthetized, and blood was collected (BD Vacutainer, BD, São Paulo, São Paulo, Brasil) from the heart ventricle for blood serum analyses. After blood centrifugation (2000 g, $20 \mathrm{~min}$ ), serum was stored in $-20^{\circ} \mathrm{C}$. Urine was also collected and stored in $-20{ }^{\circ} \mathrm{C}$ for C-terminal telopeptide of type I collagen (CTX) levels evaluation. After decapitation, mandible and femurs were re-

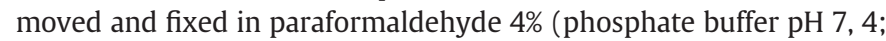
$0,1 \mathrm{M}$ ). A single examiner blind to group allocation of samples and mice performed all following analyses.

\subsection{Micro-computed tomography $(\mu C T)$}

Three-dimensional microcomputed tomography analyses of the distal femoral metaphysis were performed using a Scanco $\mu \mathrm{CT} 40$ system (Scanco Medical, Bassersdorf, Switzerland). Tomographic images were acquired at $55 \mathrm{kVp}$ and $145 \mu \mathrm{A}$ with an isotropic voxel size of $12 \mu \mathrm{m}$ and at an integration time of $300 \mathrm{~ms}$. To segment bone from nonmineralized tissue, a Gaussian noise suppression filter ( $\sigma=0.3$, support $=1$ ) was used, and global thresholds were consistent across scans per anatomical site [24].

Five right hemi-mandibles of each group were selected randomly and also scanned in a Scanco $\mu \mathrm{CT} 40$ at $70 \mathrm{kV}$ and $114 \mu \mathrm{A}$ X-ray source, 500 projections $/ 180^{\circ}$ rotation and $300 \mathrm{~ms}$ integration, and image were reconstructed with $16 \mu \mathrm{m}$ isotropic voxels. A three-dimensional (3D) volume of interest (VOI) was standardized and defined by the following points: the apex of the mesial root of the first molar (apical limit); the first molar furcation area (coronary limit); mesial of the mesial root of 


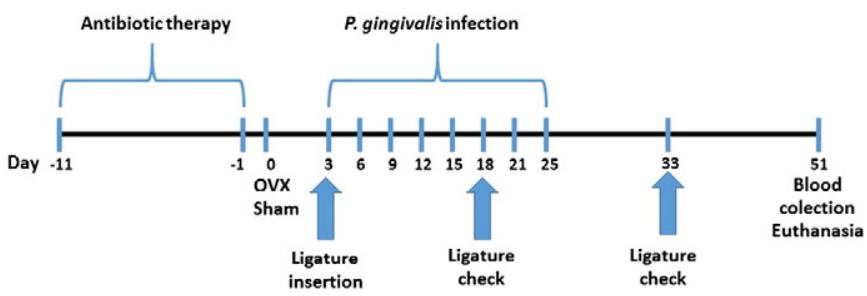

Fig. 1. Experimental design.

the second molar (posterior limit); and mesial of the mesial root of the first molar (anterior limit) [25]. For consistency between measurements, the cortex and roots were excluded. Within the VOI, a threshold of $548 \mathrm{HA} / \mathrm{ccm}$, sigma value of 0.3 , and support of 1 were applied to segment bone from soft tissue.

Bone volume fraction (BV/TV), trabecular number (Tb.N), thickness (Tb.Th) and separation (Tb.Sp) were calculated using standard methods.

\subsection{Morphometric alveolar bone loss analysis}

The left hemi-mandibles were washed in tap water to remove formalin residues, immersed in boiling water during $30 \mathrm{~min} \mathrm{[2]} \mathrm{and}$ then treated with $8 \%$ sodium hypochlorite for $4 \mathrm{~h}$. A new washing was done and the hemimandibles were defleshed mechanically.

For delimitation of the cementoenamel junction (CEJ), the specimens were stained in 1\% methylene blue [2] (Labsynth, Diadema, SP, Brazil) and had their lingual surfaces examined under stereomicroscopy. The specimens were fixed in wax in order to obtain parallelism between lingual and buccal cusps. The image at X20 magnification was digitized and the distance between CEJ and the top of lingual alveolar bone crest along the axis of each root (mesial and distal) of first molar was measured [26]. The total alveolar bone loss was considered as the mean between the mesial and distal root measurements. In this analysis, we used 9-10 samples per group.

\subsection{Enzyme-linked immunosorbent assay (ELISA)}

Blood and urine samples were thawed a room temperature. Then, serum tumor necrosis factor (TNF)- $\alpha$, interleukin (IL)-4, IL-6, IL-17 (R\&D Systems, Minneapolis, MN, USA) and osteocalcin (OC; US Biological Life Sciences, Swampscott, Massachusetts, USA); and urine CTX (US Biological Life Sciences, Swampscott, Massachusetts, USA) concentrations were determined using commercial ELISA kits, according to manufacturer's instructions. Samples from 8 animals per group were used, since in several animals we could not collect enough blood to perform all analyzes.

\subsection{Statistical analysis}

Data were analyzed using the analysis of variance (ANOVA, F) or non-parametric Kruskal-Wallis $(H)$ test $(\alpha=0.05)$. In case of statistical differences, the t-test or Newman-Student-Keuls (NSK) were used. The tests were performed using the Bioestat 2.0 (Sociedade Civil Mamirauá, Belém, PA, Brazil). Parametric data are represented in mean and standard deviation graphs, and non-parametric data are represented in box plot graphs. Medians are showed when statistical non-parametric tests were performed [27].
A

CTX

$[H=9.015, p=0.029$, NSK post hoc test $]$

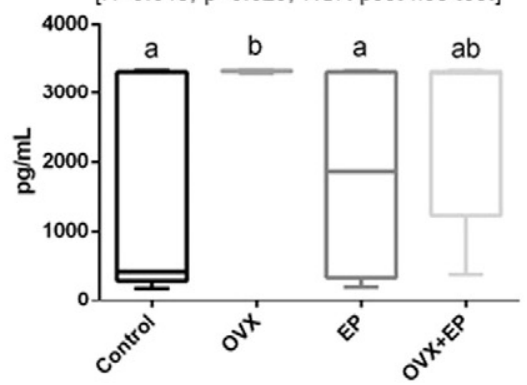

IL-6

D $[H=10.240, p=0.017$, NSK post hoc test $]$

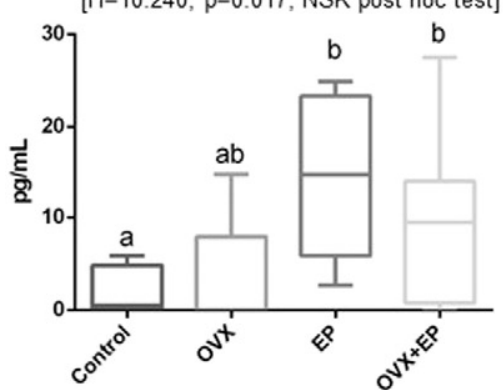

B

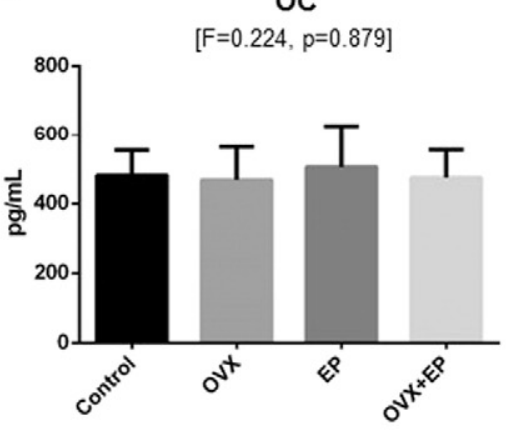

IL-17

E

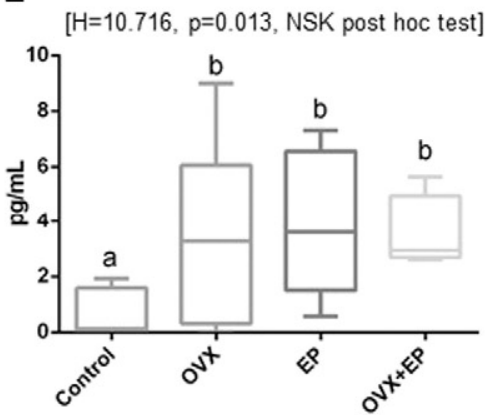

C
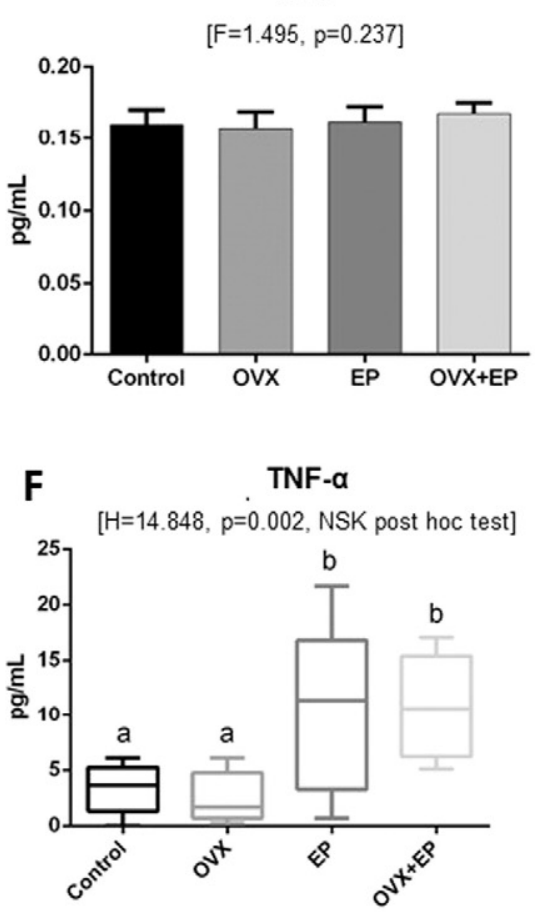

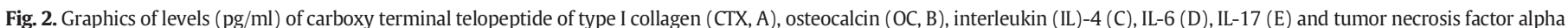

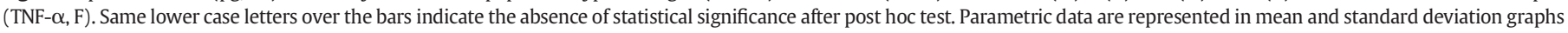

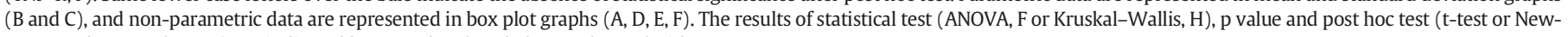
man-Student-Keuls, NSK) are indicated between brackets below each graph title. 


\section{Results}

\subsection{Body weight}

Hyperphagia associated to ovariectomy leads to increased masticatory function and consequent increase in the mechanical load applied to the alveolar bone, which could modify the direct effects of estrogen deficiency in the jaws [28]. For these reasons, in this study, we restricted the feeding of ovariectomized animals to the same amount consumed by sham animals. The percentage of weight gain over 51 days following surgery was not significantly different between the groups (Supplementary Fig. 1), confirming the success of pair-feeding.

\subsection{Detection of serum and urine cytokines}

Fifty-one days after OVX or sham-surgery, cytokine levels were measured by enzyme-linked immunosorbent assays. CTX levels were found higher in the OVX group compared to control, confirming the success of ovariectomy, but EP alone did not significantly increase urine CTX levels compared to the sham control group (Fig. 2).

All 3 experimental groups showed increased levels of IL-17 compared to control, and similar results were found regarding IL-6. Groups with EP (EP and OVX + EP) presented higher levels of TNF- $\alpha$ than groups without EP (Control and OVX). No difference was found among groups regarding serum levels of the anti-inflammatory cytokine IL-4 or OC, a marker of bone formation (Fig. 2).

\subsection{Femur micro-computed tomography}

Lower BV/TV was found in experimental groups compared to control, and the OVX group showed a significantly lower BV/TV than control, confirming the success of ovariectomy. However, the OVX + EP group showed the lowest BV/TV percentage, which was statistically different from the other groups. The OVX + EP group also showed the lowest Tb.N and Tb.Th values among groups, while presenting increased Tb.Sp (Fig. 3).

\subsection{Mandible micro-computed tomography and macroscopic measurements}

Focusing on the alveolar bone volume around the first mandibular molar, we observed that the OVX + EP group had the lowest BV/TV. Groups with EP (EP and OVX + EP) also showed increased Tb.Sp and lower Tb.Th compared to other groups. There was no difference among groups regarding Tb.N (Fig. 4).

After evaluating the bone microarchitecture by micro-tomography, the hemi-mandibles were defleshed and the linear distance between the alveolar bone crest and the cementum-enamel junction along
A

BV $/ T V$

$[H=18.768, p<0.001$, NSK post hoc test $]$

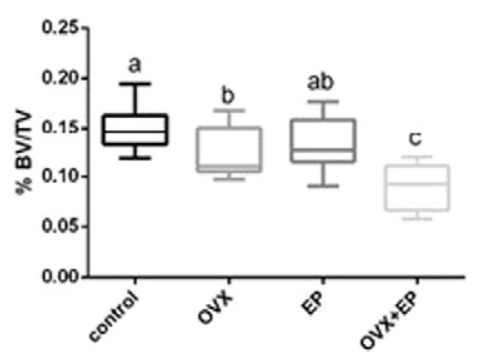

C

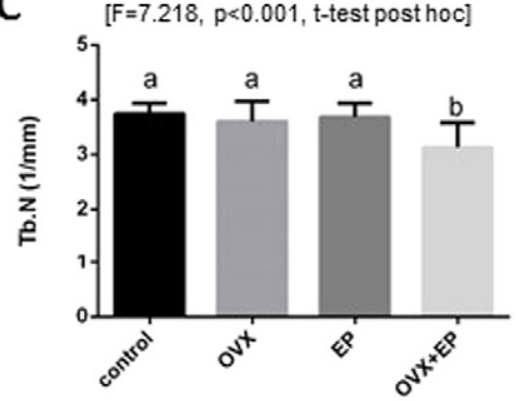

B $[H=11.542, p=0.009$, NSK post hoc test]

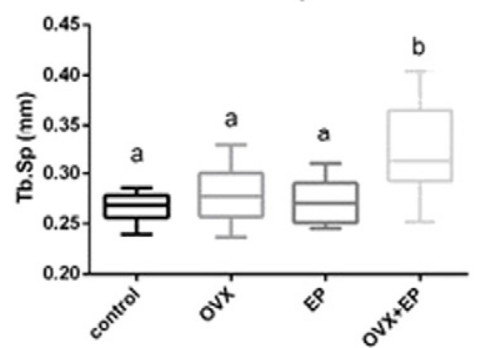

D $\quad$ Tb. Th

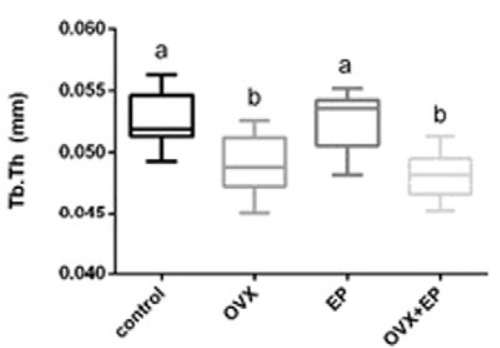

E

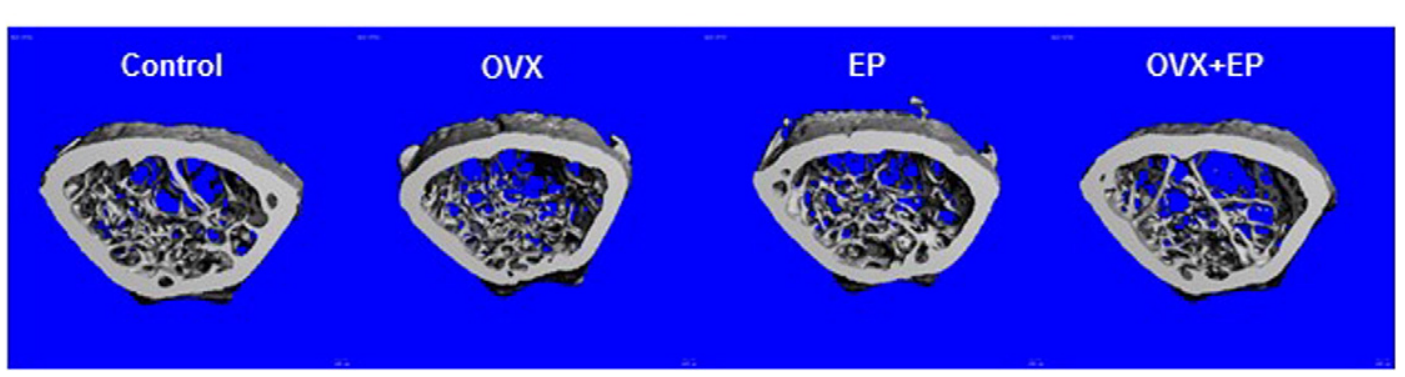

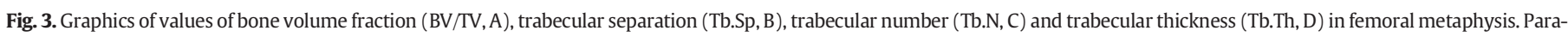

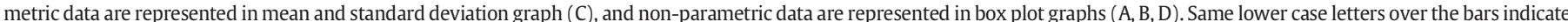

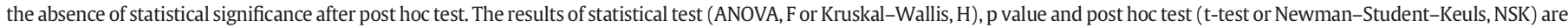

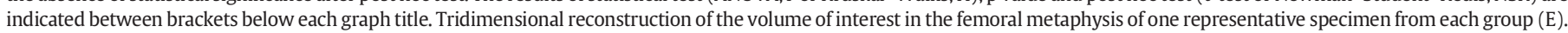
Deterioration of bone microarchitecture can be seen in the OVX + EP group. 
A

BV/TV

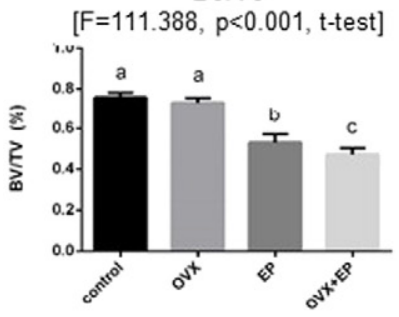

B

Tb.Sp $[F=3.715, p=0.033$, t-test $]$

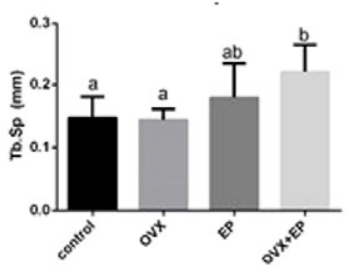

C

Tb.N

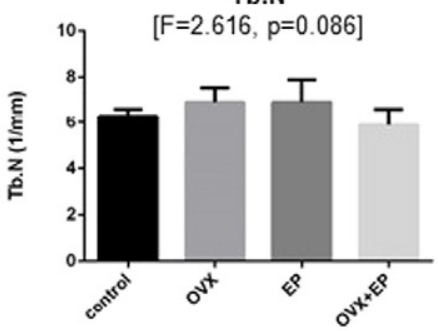

D

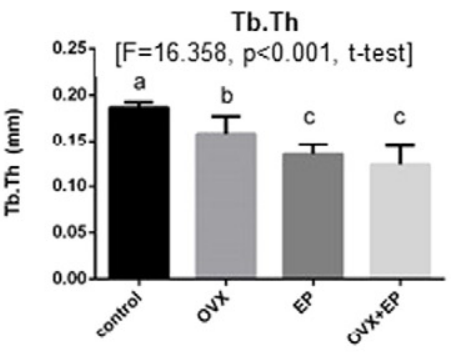

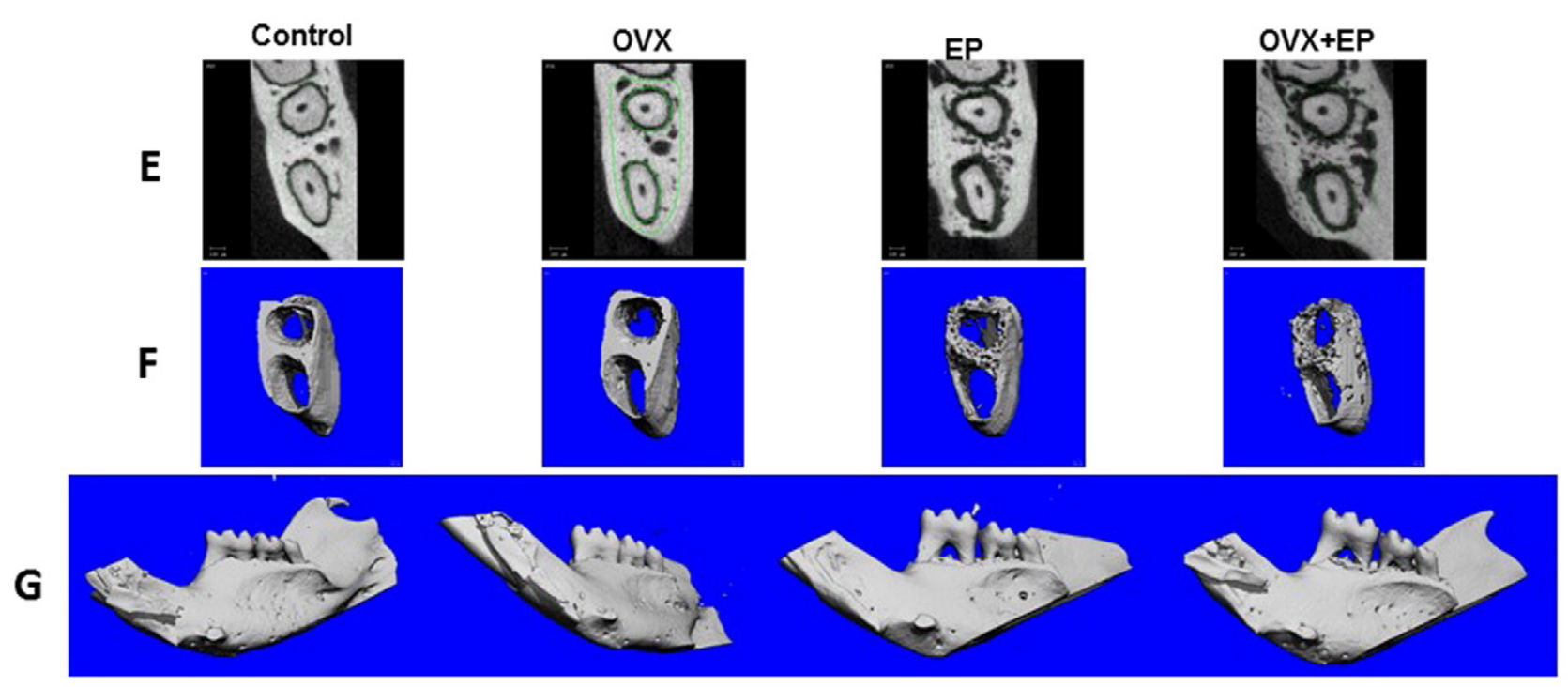

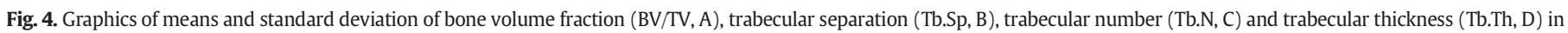

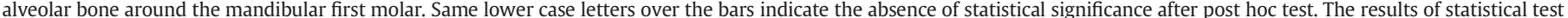

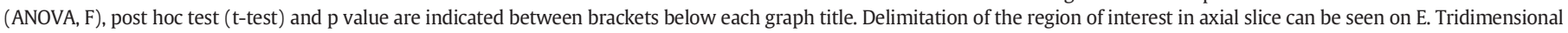

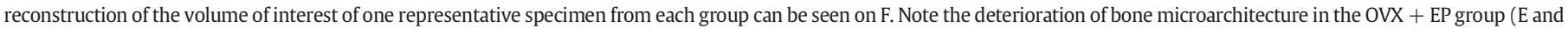
F). Tridimensional reconstruction of one representative hemi-mandible of each group to illustrate the alveolar bone loss in groups with experimental periodontitis ( $G$ ).

the two first mandibular roots was measured. Groups with EP (EP, OVX + EP) presented statistically significant higher alveolar bone loss when compared to those without EP (control, OVX) (Fig. 5). There was no difference between the EP and EP + OVX groups.

\section{Discussion}

Although osteoporosis was classified as a risk factor for periodontal disease [8-11], the relationship between periodontitis and systemic
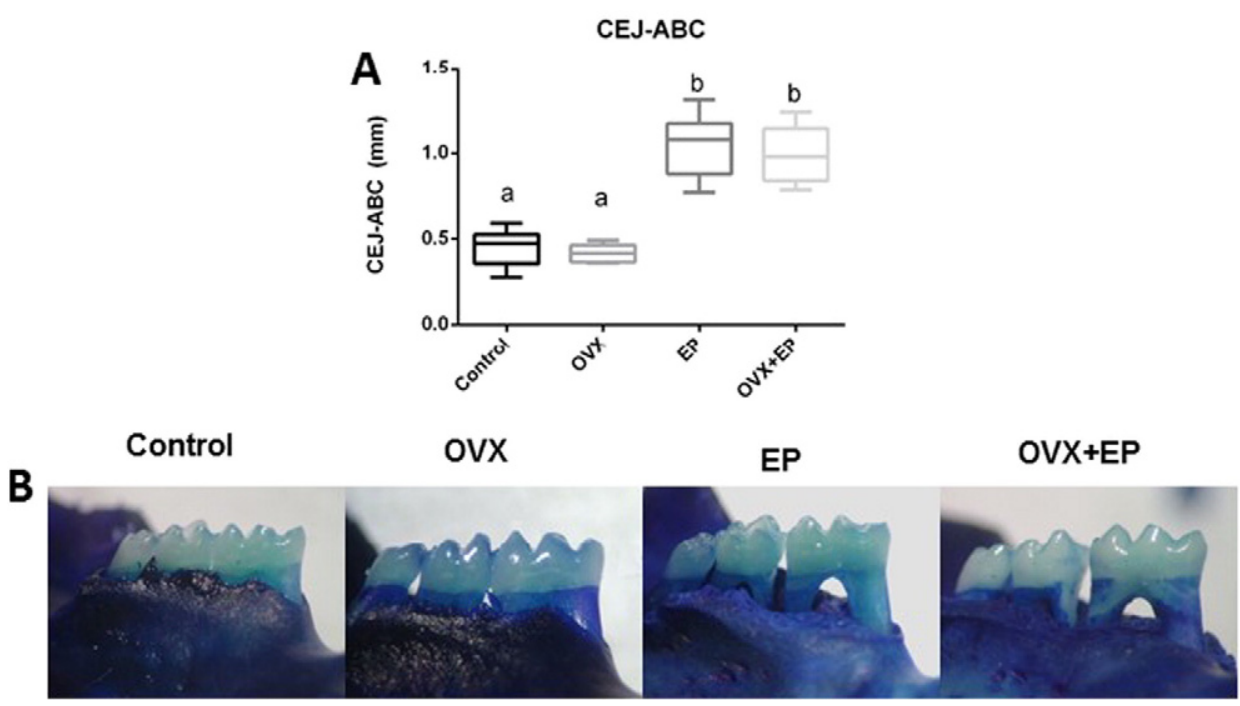

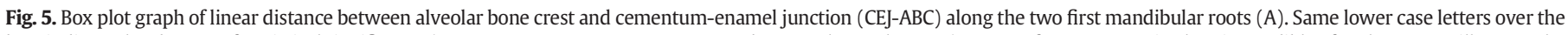

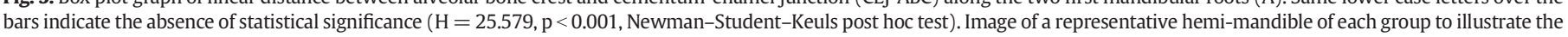
alveolar bone loss in groups with experimental periodontitis (B). 
bone loss is still controversial. Some studies have associated periodontitis and increased systemic bone loss [8-12], but a causal relationship between the two conditions is difficult to establish in prospective and retrospective clinical studies. We therefore addressed, using an experimental mouse model of combined osteoporosis and periodontal disease, whether periodontal disease influences bone homeostasis, in the context of a normal or estrogen-deficient status. Our results indicate that although experimental periodontitis alone does not significantly induce femoral bone loss, it exacerbates the bone loss associated with estrogen deficiency.

One probable mechanism involved in this effect is the production of inflammatory cytokines. EP alone induced a significant increase in IL-6, IL-17 and TNF- $\alpha$ serum levels compared to controls at end point, but did not significantly affect systemic bone parameters. OVX on the other hand led to an increase in serum IL-17 (and to a lesser extend IL-6) and urinary CTX levels, which was accompanied by a significant reduction in femoral BV/TV compared to sham controls, but it did not affect TNF- $\alpha$ serum levels at end point. A chronic elevation of TNF- $\alpha$ serum levels thus stands as the most likely mediator for the aggravation of the ovariectomy-induced systemic bone loss in OVX + EP mice. Among the many factors involved in the pathophysiology of bone loss, TNF- $\alpha$ has a central role. It stimulates osteoclastogenesis, suppresses the recruitment of osteoblasts from progenitor cells, induces resistance to 1,25-dihydroxyvitamin D3 and inhibits the expression of matrix protein genes [29]. TNF- $\alpha$ is also known to contribute to periodontal damage by amplifying inflammatory reactions [30] and stimulating the expression and activation of matrix metalloproteinases that degrade connective tissue matrix [31]. In patients with periodontitis, TNF- $\alpha$ concentrations in serum samples and gingival tissue biopsies are higher compared to healthy individuals [32]. In addition, blockade of TNF- $\alpha$ action leads to significantly anti-inflammatory properties and decreases alveolar bone loss in rats with EP [33].

Our experimental setting also allowed us to investigate the effect of estrogen deficiency on the alveolar bone supporting teeth. We show that 51 days after surgery, OVX mice had lower mandibular Tb.Th than control, although alveolar BV/TV, trabecular space and number were not significantly affected, in contrast to results from Macari et al. [34], who evaluated the interradicular septum of maxillary first molar. These different results could be explained by the different mechanical forces that are applied to the mandible and maxilla, that can partially prevent cancellous bone loss in OVX rats $[28,35]$. Indeed, the effect of estrogen deficiency on rat jaws is controversial, and may be dependent on difference in the duration of estrogen deficiency and the region analyzed [36]. While some authors observed no jaw alterations resulting from ovariectomy [36-38], others [39-42] found a negative effect of estrogen deficiency in several jaw regions.

EP as expected induced alveolar bone loss, and the combination $\mathrm{EP}+\mathrm{OVX}$ aggravated the deterioration in alveolar bone microarchitecture compared to mice with EP alone, as shown by a significantly lower BV/TV around the first molar, in agreement with other studies in rats [43] and mice [44]. These results suggest that estrogen deficiency can exacerbate the severity of alveolar bone deterioration, via mechanisms to be identified. We did not find significant difference in the distance between cementum enamel junction and alveolar bone crest between mice without EP (no difference between OVX and control groups) or with EP (no difference between EP and OVX + EP groups) at the 51 day end point, in agreement with another study in mice [45]. These results suggest that estrogen deficiency may not directly affect alveolar bone height, although a recent study showed increased alveolar bone crest loss in OVX mice (without EP) compared to control animals, 15 and 30 days after OVX [34].

\section{Conclusion}

Our results in mice provide experimental evidence that periodontitis could be a risk factor for systemic bone loss, especially in women with estrogen deficiency, and possibly in individuals characterized by proinflammatory conditions, which are prevalent in our aging population. They support a previously reported association study suggesting that older individuals with both osteoporosis and periodontitis are at greater risk for future fractures than those with either osteoporosis or periodontitis alone [13]. Further clinical investigations are thus warranted to confirm this association and propose adapted prophylactic and curative therapies. The effect of osteoporosis on the onset and progression of periodontitis is also to be further explored in aging individuals to develop therapeutic options limiting alveolar bone loss.

Supplementary data to this article can be found online at http://dx. doi.org/10.1016/j.bone.2015.11.014.

\section{Acknowledgment}

This project was funded by the São Paulo Research Foundation (FAPESP 2012/23825-2 (ALA) and 2013/00718-9 (DRCC) and institutional seed funds from the Vanderbilt Department of Medicine/Center for Bone Biology (FE). The authors declare no conflict of interest.

\section{References}

[1] United Nations, Department of Economic and Social Affairs, Population Division, World Population Ageing 2013, 2013 (ST/ESA/SER.A/348).

[2] A.L. Anbinder, A. Prado Mde, M. Spalding, I. Balducci, Y.R. Carvalho, R.F. da Rocha, Estrogen deficiency and periodontal condition in rats: a radiographic and macroscopic study, Braz. Dent. J. 17 (3) (2006) 201-207.

[3] H.K. Genant, C. Cooper, G. Poor, I. Reid, G. Ehrlich, J. Kanis, et al., Interim report and recommendations of the World Health Organization Task-Force for Osteoporosis, Osteoporos. Int. 10 (4) (1999) 259-264.

[4] J.A. Cauley, Public health impact of osteoporosis, J. Gerontol. A Biol. Sci. Med. Sci. 68 (10) (2013) 1243-1251

[5] The American Academy of Periodontology, Glossary of Periodontal Terms, 4th ed., 2001 (Chicago, p. 53p).

[6] P.I. Eke, B.A. Dye, L. Wei, G.D. Slade, G.O. Thornton-Evans, W.S. Borgnakke, et al., Update on prevalence of periodontitis in adults in the United States: NHANES 2009 to 2012, J. Periodontol. 86 (5) (2015) 611-622.

[7] M. Martínez-Maestre, C. González-Cejudo, G. Machuca, R. Torrejón, C. CasteloBranco, Periodontitis and osteoporosis: a systematic review, Climacteric 13 (6) (2010) 523-529.

[8] R.J. Genco, W.S. Borgnakke, Risk factors for periodontal disease, Periodontol. 62 (1) (2013) 59-94.

[9] V. Esfahanian, M.S. Shamami, Relationship between osteoporosis and periodontal disease: review of the literature, J. Dent. (Tehran) 9 (4) (2012) 256-264.

[10] R. Guiglia, O. Di Fede, L. Lo Russo, D. Sprini, G.B. Rini, G. Campisi, Osteoporosis, jawbones and periodontal disease, Med. Oral Patol. Oral Cir. Bucal 18 (1) (2013) e93-e99.

[11] L.C. Kuo, A.M. Poison, T. Kang, Associations between periodontal diseases and systemic diseases: a review of the inter-relationships and interactions with diabetes, respiratory diseases, cardiovascular diseases and osteoporosis, Public Health 122 (4) (2008) 417-433.

[12] G. Pizzo, R. Guiglia, L. Lo Russo, G. Campisi, Dentistry and internal medicine: from the focal infection theory to the periodontal medicine concept, Eur. J. Intern. Med. 21 (6) (2010) 496-502.

[13] G.R. Persson, J. Berglund, R.E. Persson, S. Renvert, Prediction of hip and hand fractures in older persons with or without a diagnosis of periodontitis, Bone 48 (3) (2011) 552-556.

[14] R.M. Moraes, G.M. Lima, F.E. Oliveira, A.C. Valim, R.C. Pereira, L.D. Oliveira, et al. Exenatide and sitagliptin decrease the expression of II $1 \mathrm{~b}, \mathrm{Mmp} 9$ and Nos2, but does not reduce alveolar bone loss in rats with periodontitis, J. Periodontol. (2015) 1-15 [Epub ahead of print].

[15] D.T. Graves, J. Kang, O. Andriankaja, K. Wada, C. Rossa, Animal models to study hostbacteria interactions involved in periodontitis, Front. Oral Biol. 15 (2012) 117-132.

[16] P.J. Baker, R.T. Evans, D.C. Roopenian, Oral infection with Porphyromonas gingivalis and induced alveolar bone loss in immunocompetent and severe combined immunodeficient mice, Arch. Oral Biol. 39 (12) (1994) 1035-1040.

[17] R.K.H. Orth, N.M. O'Brien-Simpson, S.G. Dashper, E.C. Reynolds, Synergistic virulence of Porphyromonas gingivalis and Treponema denticola in a murine periodontitis model, Mol. Oral Microbiol. 26 (4) (2011) 229-240.

[18] R.S. de Molon, E.D. de Avila, A.V. Nogueira, J.A. de Souza, M.J. Avila-Campos, C.R. de Andrade, et al., Evaluation of the host response in various models of induced periodontal disease in mice, J. Periodontol. (2013).

[19] K. Saadi-Thiers, O. Huck, P. Simonis, P. Tilly, J.E. Fabre, H. Tenenbaum, et al., Periodontal and systemic responses in various mice models of experimental periodontitis: respective roles of inflammation duration and Porphyromonas gingivalis infection, J. Periodontol. 84 (3) (2013) 396-406.

[20] C.H. Li, S. Amar, Morphometric, histomorphometric, and microcomputed tomographic analysis of periodontal inflammatory lesions in a murine model, J. Periodontol. 78 (6) (2007) 1120-1128. 
[21] J. Lin, L. Bi, X. Yu, T. Kawai, M.A. Taubman, B. Shen, et al., Porphyromonas gingivalis exacerbates ligature-induced, RANKL-dependent alveolar bone resorption via differential regulation of toll-like receptor 2 (TLR2) and TLR4, Infect. Immun. 82 (10) (2014) 4127-4134.

[22] F. Montagner, R.C. Jacinto, F.G. Correa Signoretti, V. Scheffer de Mattos, F.S. Grecca B.P. Gomes, Beta-lactamic resistance profiles in Porphyromonas, Prevotella, and Parvimonas species isolated from acute endodontic infections, J. Endod. 40 (3) (2014) 339-344

[23] S.R. Orrico, G. Giro, D. Goncalves, L. Takayama, R.M. Pereira, Influence of the period after ovariectomy on femoral and mandibular bone density and on induced periodontal disease, J. Periodontol. 78 (1) (2007) 164-169.

[24] M.L. Bouxsein, S.K. Boyd, B.A. Christiansen, R.E. Guldberg, K.J. Jepsen, R. Müller Guidelines for assessment of bone microstructure in rodents using microcomputed tomography, J. Bone Miner. Res. 25 (7) (2010 Jul) 1468-1486.

[25] M.R. Guimarães, L.S. Coimbra, S.G. de Aquino, L.C. Spolidorio, K.L. Kirkwood, C. Rossa Jr., Potent anti-inflammatory effects of systemically administered curcumin modulate periodontal disease in vivo, J. Periodontal Res. 46 (2) (2011 Apr) 269-279.

[26] G.E. Barrella, I.B. Suffredini, F.V. Ribeiro, F.R. Cirano, S.P. Pimentel, Evaluation of the effect of an organic extract obtained from Ipomoea alba L. on experimental periodontitis in rats, Braz. Oral Res. 26 (2) (2012) 158-164.

[27] T.L. Weissgerber, N.M. Milic, S.J. Winham, V.D. Garovic, Beyond bar and line graphs: time for a new data presentation paradigm, PLoS Biol. 13 (4) (2015), e1002128.

[28] D. Zaffe, C. Paganelli, D. Cocchi, Induction and pharmacological treatment of ora osteopenia in rats, Minerva Stomatol. 48 (3) (1999) 45-62.

[29] M.S. Nanes, Tumor necrosis factor-alpha: molecular and cellular mechanisms in skeletal pathology, Gene 321 (2003) 1-15.

[30] A. Di Benedetto, I. Gigante, S. Colucci, M. Grano, Periodontal disease: linking the primary inflammation to bone loss, Clin. Dev. Immunol. 2013 (2013) 503754

[31] D.T. Graves, T. Oates, G.P. Garlet, Review of osteoimmunology and the host response in endodontic and periodontal lesions, J. Oral Microbiol. 3 (2011).

[32] R. Górska, H. Gregorek, J. Kowalski, A. Laskus-Perendyk, M. Syczewska, K. Madaliński, Relationship between clinical parameters and cytokine profiles in in flamed gingival tissue and serum samples from patients with chronic periodontitis, J. Clin. Periodontol. 30 (12) (2003) 1046-1052.
[33] D.C. Gonçalves, R.C. Evangelista, R.R. da Silva, M.J. Santos, F.S. Silva, K.S. Aragão, et al., Infliximab attenuates inflammatory osteolysis in a model of periodontitis in Wistar rats, Exp. Biol. Med. (Maywood) 239 (4) (2014) 442-453.

[34] S. Macari, L.F. Duffles, C.M. Queiroz-Junior, M.F. Madeira, G.J. Dias, M.M. Teixeira, et al., Oestrogen regulates bone resorption and cytokine production in the maxillae of female mice, Arch. Oral Biol. 60 (2) (2015) 333-341.

[35] B.Y. Lin, W.S. Jee, M.M. Chen, Y.F. Ma, H.Z. Ke, X.J. Li, Mechanical loading modifies ovariectomy-induced cancellous bone loss, Bone Miner. 25 (3) (1994) 199-210.

[36] C.M. Esteves, R.M. Moraes, F.C. Gomes, M.S. Marcondes, G.M. Lima, A.L. Anbinder, Ovariectomy-associated changes in interradicular septum and in tibia metaphysis in different observation periods in rats, Pathol. Res. Pract. 211 (2) (2015) 125-129.

[37] J.M. Teófilo, A.C. Azevedo, S.O. Petenusci, R. Mazaro, T.L. Lamano-Carvalho, Comparison between two experimental protocols to promote osteoporosis in the maxilla and proximal tibia of female rats, Pesqui. Odontol. Bras. 17 (4) (2003) 302-306.

[38] Y. Moriya, K. Ito, S. Murai, Effects of experimental osteoporosis on alveolar bone loss in rats, J. Oral Sci. 40 (4) (1998) 171-175.

[39] M. Tanaka, S. Ejiri, E. Toyooka, S. Kohno, H. Ozawa, Effects of ovariectomy on trabecular structures of rat alveolar bone, J. Periodontal Res. 37 (2) (2002) 161-165.

[40] T. Hara, T. Sato, M. Oka, S. Mori, H. Shirai, Effects of ovariectomy and/or dietary calcium deficiency on bone dynamics in the rat hard palate, mandible and proximal tibia, Arch. Oral Biol. 46 (5) (2001) 443-451.

[41] G. Jiang, H. Matsumoto, A. Fujii, Mandible bone loss in osteoporosis rats, J. Bone Miner. Metab. 21 (6) (2003) 388-395.

[42] P.M. Duarte, P.F. Goncalves, A.W. Sallum, E.A. Sallum, M.Z. Casati, F. Humberto Nociti Jr., Effect of an estrogen-deficient state and its therapy on bone loss resulting from an experimental periodontitis in rats, J. Periodontal Res. 39 (2) (2004) 107-110.

[43] J. Dai, Y. Ma, M. Shi, Z. Cao, Y. Zhang, R.J. Miron, Initial changes in alveolar bone volume for sham-operated and ovariectomized rats in ligature-induced experimental periodontitis, Clin. Oral Investig. (2015).

[44] M. Kobayashi, C. Matsumoto, M. Hirata, T. Tominari, M. Inada, C. Miyaura, The correlation between postmenopausal osteoporosis and inflammatory periodontitis regarding bone loss in experimental models, Exp. Anim. 61 (2) (2012) 183-187.

[45] D. Nebel, G. Bratthall, G. Warfvinge, B.O. Nilsson, Effects of ovariectomy and aging on tooth attachment in female mice assessed by morphometric analysis, Acta Odontol. Scand. 67 (1) (2009) 8-12. 